\title{
Knockdown of JARID2 inhibits the proliferation and invasion of ovarian cancer through the PI3K/Akt signaling pathway
}

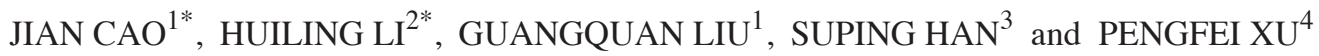 \\ ${ }^{1}$ Department of Obstetrics and Gynecology, Obstetrics and Gynecology Hospital Affiliated to Nanjing Medical University, \\ Nanjing, Jiangsu 210004; ${ }^{2}$ Department of Obstetrics and Gynecology, Peking University Third Hospital, Beijing 100191; \\ ${ }^{3}$ Department of Obstetrics and Gynecology, The First Affiliated Hospital of Nanjing Medical University, Nanjing, \\ Jiangsu 210029; ${ }^{4}$ Nanjing Maternal and Child Health Institute, Obstetrics and Gynecology Hospital Affiliated to \\ Nanjing Medical University, Nanjing, Jiangsu 210004, P.R. China
}

Received September 24, 2016; Accepted May 26, 2017

DOI: $10.3892 / \mathrm{mmr} .2017 .7024$

\begin{abstract}
Protein Jumonji (JARID2), a member of the family of JmjC domain-containing proteins, has been reported to serve an important role in tumor growth and metastasis. However, the expression pattern and role of JARID2 in ovarian cancer remains unclear. Therefore, in the present study, the role of JARID2 in ovarian cancer was investigated, as well as the underlying mechanisms. The results of the present study demonstrated that the expression of JARID2 is upregulated in human ovarian cancer cell lines. Furthermore, downregulation of JARID2 significantly suppressed proliferation, migration, invasion and epithelial-mesenchymal transition in human ovarian cancer cells. Mechanistically, downregulation of JARID2 decreased the protein expression levels of phosphorylated phosphoinositide 3-kinase (PI3K) and protein kinase B (Akt) in ovarian cancer cells. In conclusion the observations suggested that knockdown of JARID2 inhibited proliferation, migration and invasion in vitro through the inactivation of the PI3K/Akt signaling pathway. Therefore, JARID2 may represent a potential therapeutic target for the treatment of ovarian cancer.
\end{abstract}

\section{Introduction}

Ovarian cancer is the most common cause of mortality among women in the world and its incidence has been rising worldwide over the past few decades (1). Despite extensive efforts to

Correspondence to: Dr Suping Han, Department of Obstetrics and Gynecology, The First Affiliated Hospital of Nanjing Medical University, 300 Guangzhou Road, Nanjing, Jiangsu 210029, P.R. China E-mail: suping_han@163.com

Dr Pengfei Xu, Nanjing Maternal and Child Health Institute, Obstetrics and Gynecology Hospital Affiliated to Nanjing Medical University, 123 Mo'chou Road, Nanjing, Jiangsu 210004, P.R. China

E-mail: xupeng_feigyn@126.com

*Contributed equally

Key words: protein Jumonji, ovarian cancer, proliferation, invasion improve the diagnosis and treatment of ovarian cancer, limited progress has been made (2-4). The majority of patients with ovarian cancer are diagnosed at advanced stages due to the subtle symptoms at early stages of ovarian carcinogenesis and the 5-year survival rate of patients with ovarian cancer is $<30 \%(5,6)$. Therefore, there is a requirement to understand the underlying mechanisms of ovarian cancer progression for the treatment of ovarian cancer.

Protein Jumonji (JARID2) is a member of the family of JmjC domain-containing proteins that remove methyl residues from methylated lysine 4 on histone H3 lysine 4 (H3K4) (7). JARID2 is crucial for the maintenance of pluripotency and differentiation in embryonic stem cells (ESCs) (8). JARID2 depletion has been reported to cause pronounced defects during ESC differentiation $(9,10)$. In addition, aberrant expression of JARID2 has been reported in several types of cancers, and JARID2 may promote tumor growth and metastasis (11-13). For example, Lei et al (14) reported that JARID2 expression was increased in hepatocellular carcinoma (HCC) tissues compared with in adjacent non-tumor liver tissues, and upregulation of JARID2 promoted HCC cell proliferation, migration and invasion in vitro, and metastasis in vivo. However, the expression pattern and role of JARID2 in ovarian cancer remains unclear. Therefore, the present study investigated the role of JARID2 in ovarian cancer and the associated underlying mechanisms. The results demonstrated that knockdown of JARID2 inhibited the proliferation, migration and invasion of ovarian cancer cells in vitro. Therefore, JARID2 may represent a potential therapeutic target for the treatment of ovarian cancer.

\section{Materials and methods}

Cell culture. A total of three human ovarian cancer cell lines (SKOV3, 3AO and OVCAR3) and a human normal ovarian surface epithelial cell line (HOSEpiC) were purchased from the American Type Culture Collection (Manassas, VA, USA). All cells were maintained in Dulbecco's Modified Eagle's Medium (DMEM; Gibco; Thermo Fisher Scientific, Inc., Waltham, MA USA) supplemented with $10 \%(\mathrm{v} / \mathrm{v})$ fetal bovine serum (FBS; Gibco; Thermo Fisher Scientific, Inc.), 100 U/ml streptomycin 
and $100 \mathrm{U} / \mathrm{ml}$ penicillin (Gibco; Thermo Fisher Scientific, Inc.) at $37^{\circ} \mathrm{C}$ in a humidified atmosphere containing $5 \% \mathrm{CO}_{2}$.

Reverse transcription-quantitative polymerase chain reaction (RT-qPCR). Total RNA was extracted from ovarian cancer cells using TRIzol reagent (Invitrogen; Thermo Fisher Scientific, Inc.) and the cDNA was reverse transcribed using the EasyScript First-Strand cDNA Synthesis SuperMix kit (Invitrogen; Thermo Fisher Scientific, Inc.) according to the manufacturer's protocol. qPCR reactions were performed on the Bio-Rad iQ5 real-time thermal cyclers using SYBR Premix Ex Taq ${ }^{\mathrm{TM}}$ II kit (Takara Biotechnology Co., Ltd., Dalian, China). The following PCR primers were used: JARID2 forward, 5'-GACACCAAA CCCAATCACCAC-3' and reverse, 5'-GTTCAACCTGCC ACTGACCTT-3'; epithelial (E)-cadherin forward, 5'-TACACT GCCCAGGAGCCAGA-3' and reverse, 5'-TGGCACCAGTGT CCGGATTA-3'; neural (N)-cadherin forward, 5'-TTTGAT GGAGGTCTCCTAACACC-3' and reverse, 5'-ACGTTTAAC ACGTTGGAAATGTG-3'; and $\beta$-actin forward, 5'-TTAGTT GCGTTACACCCTTTC-3' and reverse, 5'-ACCTTCACCGTT CCAGTTT-3'. The PCR cycling program was as follows: $95^{\circ} \mathrm{C}$ for $5 \mathrm{~min}$, followed by 35 cycles of $95^{\circ} \mathrm{C}$ for $20 \mathrm{sec}, 59^{\circ} \mathrm{C}$ for $20 \mathrm{sec}$ and $72^{\circ} \mathrm{C}$ for $20 \mathrm{sec}$, and a final extension at $72^{\circ} \mathrm{C}$ for 5 min. $\beta$-actin was used as the internal reference gene. Then the PCR products were separated by agarose gel electrophoresis to confirm successful amplification. The relative expression levels were calculated using the $2^{-\Delta \Delta C q}$ method (15).

Western blotting. Total protein was extracted from ovarian cancer cells using radioimmunoprecipitation assay buffer containing phosphatase and protease inhibitors (Sigma-Aldrich; Merck KGaA, Darmstadt, Germany). The protein concentration was then determined using the Bradford method. Protein lysates $(30 \mu \mathrm{g})$ were separated by $10 \%$ SDS-PAGE and transferred onto polyvinylidene difluoride membranes (EMD Millipore, Billerica, MA, USA). Then, the membrane was blocked with $5 \%$ non-fat milk in TBS with Tween-20 [TBST; $10 \mathrm{mM}$ Tris- $\mathrm{HCl}$ (pH of 7.5), $150 \mathrm{mM} \mathrm{NaCl}$ and $0.05 \%$ Tween-20] for $1 \mathrm{~h}$ at room temperature, followed by incubation with primary antibodies anti-JARID2 (1:3,000; cat. no. SAB2105079; Sigma-Aldrich; Merck KGaA), and E-cadherin (1:3,000; cat. no. sc-21791), N-cadherin $(1: 2,000$; cat. no. sc-53488), PI3K (1:2,000; cat. no. sc-365290), phosphorylated-phosphoinositide 3-kinase (p-PI3K; 1:2,500; cat. no. sc-293115), protein kinase B (Akt; 1:3,000; sc-5298), p-Akt (1:2,500; cat. no. sc-52940) and GAPDH (1:2,000; cat. no. sc-47724), from Santa Cruz Biotechnology, Inc. (Dallas, TX, USA) overnight at $4^{\circ} \mathrm{C}$. Subsequently, the membranes were incubated with horseradish peroxidase-conjugated secondary antibody (1:2,500; cat. no. sc-2005; Santa Cruz Biotechnology, Inc.) at room temperature for $1 \mathrm{~h}$. Bound antibodies were detected using enhanced chemiluminescence (Pierce; Thermo Fisher Scientific, Inc.). Relative protein levels of p-PI3K and p-Akt were quantified using Image-Pro Plus software (version 6.0; Media Cybernetics, Inc., Rockville, MD, USA).

RNA interference and cell transfection. Small interfering RNA against JARID2 (si-JARID2, 5'-AGGAAGAGGAGG AGGACAA-3') and its negative control (si-NC, 5'-GAGUGG GUCUGGGUCUUCCCGUAGA-3') were synthesized by
Guangzhou RiboBio Co., Ltd. (Guangzhou, China). SKOV3 cells were seeded at a density of $1 \times 10^{5}$ cells/well in each cell of a 24-well microplate, grown for $24 \mathrm{~h}$ to reach $40 \%$ confluence, and then transfected with si-JARID2 or si-NC using $4 \mu \mathrm{l}$ Lipofectamine 2000 transfection reagent (Invitrogen; Thermo Fisher Scientific, Inc.) for $24 \mathrm{~h}$, according to the manufacturer's protocol. The efficacy of overexpression was confirmed by RT-qPCR and western blot analysis.

Cell proliferation assay. Cell proliferation was measured using the MTT assay (Sangon Biotech Co., Ltd., Shanghai, China). SKOV3 cells were seeded at a density of $3 \times 10^{4}$ cells/well into 96-well culture plates and cultured for 1-4 days following transfection. At each time point, $20 \mu \mathrm{l}$ PBS containing $5 \mathrm{mg} / \mathrm{ml}$ MTT/well was added and incubated for $4 \mathrm{~h}$ at $37^{\circ} \mathrm{C}$. The medium was removed, and dimethyl sulfoxide was added to each well. The absorbance was measured at $490 \mathrm{~nm}$ with a microplate reader (Bio-Rad, Laboratories, Inc., Hercules, CA, USA).

Transwell migration and invasion assays. Cell migration and invasion were detected using the Transwell chamber assay. For the cell migration assay, transfected ovarian cancer cells ( $1 \times 10^{5}$ cells/chamber) in $100 \mu \mathrm{l}$ serum-free media were plated into the upper chamber, while $500 \mu 1$ DMEM medium with $10 \%$ FBS was added into the lower compartment. Following incubation for $24 \mathrm{~h}$ at $37^{\circ} \mathrm{C}$, the non-migratory cells were removed with cotton swabs from the upper surface of the membrane. The cells on the lower surface of the membrane were fixed with $4 \%$ paraformaldehyde, stained with $0.1 \%$ crystal violet at room temperature for $10 \mathrm{~min}$, and then counted under a light microscope (magnification, x100). For the invasion assay, the procedures were the same as the migration assay except the chamber was pre-coated with Matrigel (BD Biosciences, Franklin Lakes, NJ USA).

Statistical analysis. All statistical analyses were conducted using SPSS software (version 13.0; SPSS, Inc., Chicago, IL, USA). Statistical significance was analyzed with one-way factorial analysis of variance followed by Tukey's post hoc test or Student's two-tailed t-test. $\mathrm{P}<0.05$ was considered to indicate a statistically significant difference.

\section{Results}

JARID2 is highly expressed in ovarian cancer cell lines. RT-qPCR was performed to detect the level of JARID2 mRNA expression in human ovarian cancer cell lines. It was observed that the mRNA expression levels of JARID2 were increased in human ovarian cancer cell lines compared with the control group (Fig. 1A). Furthermore, the protein expression of JARID2 in human ovarian cancer cell lines was examined by western blotting. All cell lines investigated exhibited increased JARID2 protein expression levels in human ovarian cancer cell lines compared with the control HOSEpiC cell line (Fig. 1B).

Downregulation of JARID2 inhibits the proliferation of ovarian cancer cells. In order to investigate the role of JARID2 in ovarian cancer carcinogenesis, a si-JARID2 was introduced into ovarian cancer cells. The results of the RT-qPCR analysis demonstrated that the mRNA expression of JARID2 was 

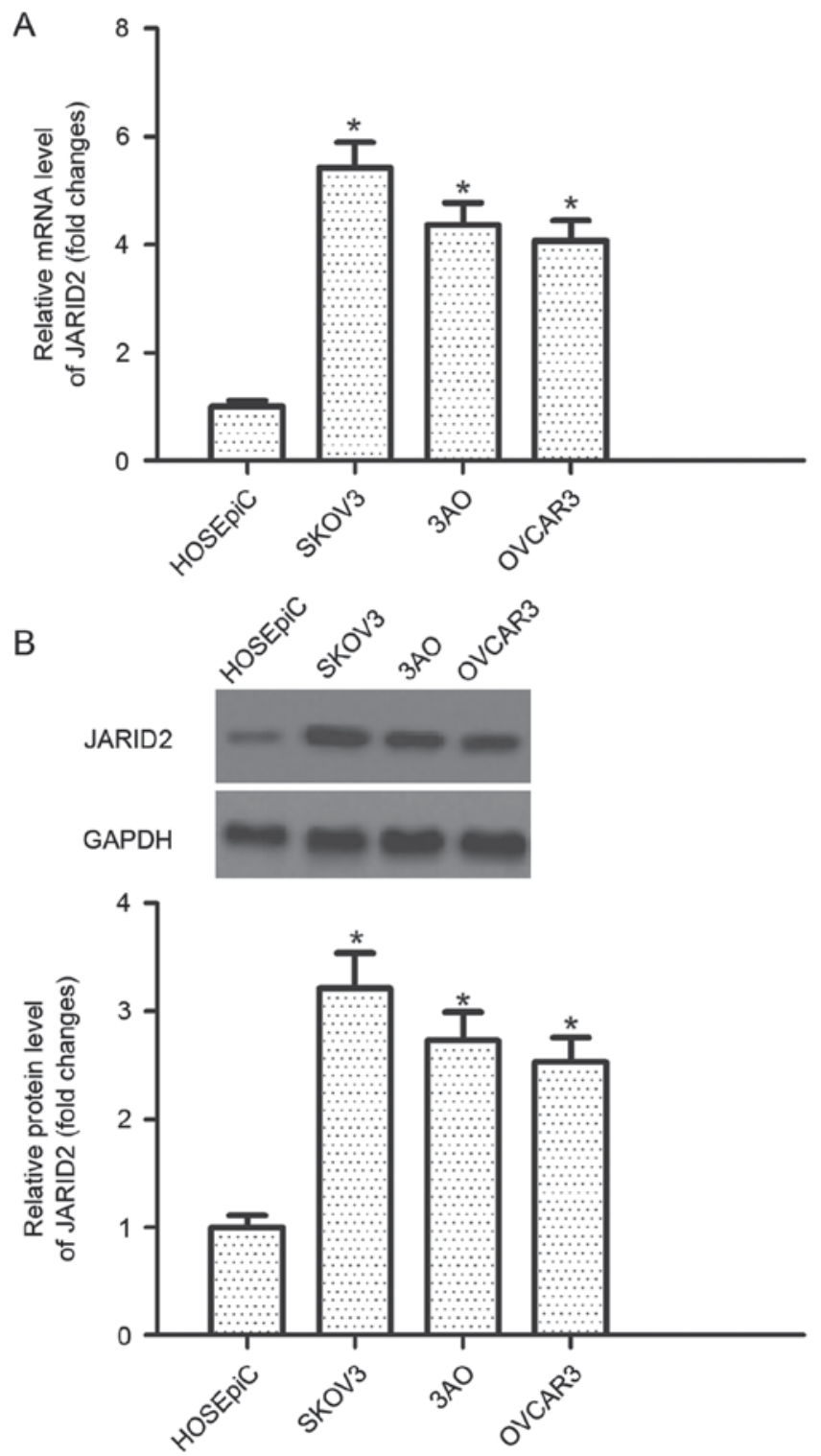

Figure 1. JARID2 is expressed at high levels in ovarian cancer cell lines. (A) mRNA expression levels of JARID2 were evaluated using the reverse transcription-quantitative polymerase chain reaction in human ovarian cancer cell lines. (B) Protein expression of JARID2 in human ovarian cancer cell lines was detected using western blotting. ${ }^{\mathrm{P}}<0.05$ vs. HOSEpiC cells. JARID2, protein Jumonji.

significantly decreased in ovarian cancer cells infected with si-JARID2 compared with the si-NC group (Fig. 2A). Western blot analysis indicated that si-JARID2 also downregulated the protein expression of JARID2 in ovarian cancer cells compared with the si-NC group (Fig. 2B). Then, the effect of JARID2 on ovarian cancer cell proliferation was examined using the MTT assay. As presented in Fig. 2C, downregulation of JARID2 significantly suppressed the proliferation of ovarian cancer cells, as compared with the si-NC group.

Downregulation of JARID2 inhibits the migration and invasion of ovarian cancer cells. The effect of JARID2 on the migration and invasion of ovarian cancer cells was investigated. Compared with the si-NC group, SKOV3 cells transfected with si-JARID2 exhibited a significant reduction in cell migration (Fig. 3A). Furthermore, the results of the
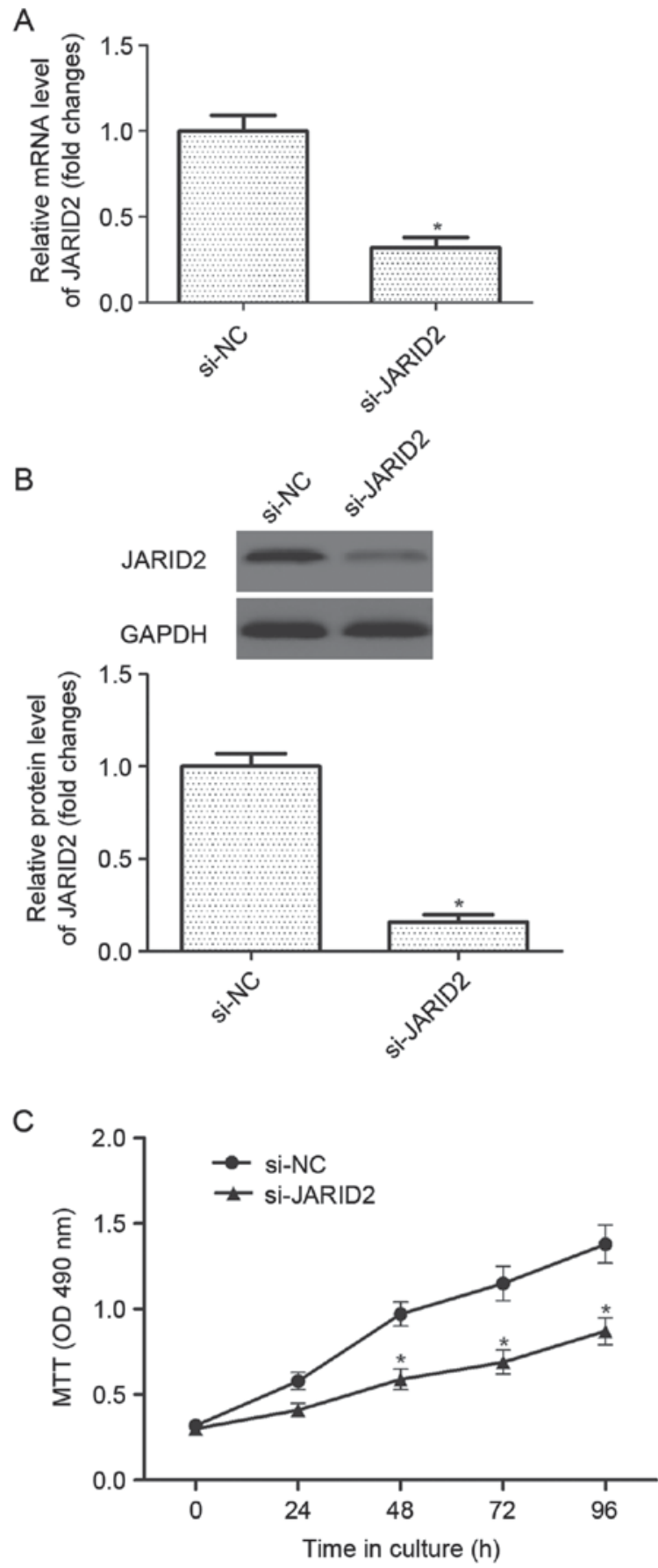

Figure 2. Downregulation of JARID2 inhibits the proliferation of ovarian cancer cells. SKOV3 cells were transfected with si-JARID2 or si-NC for $24 \mathrm{~h}$. (A) mRNA expression level of JARID2 was evaluated using the reverse transcription-quantitative polymerase chain reaction. (B) Protein expression of JARID2 was detected using western blotting. (C) Cell proliferation was detected using the MTT assay. All the experiments were repeated at least three times. ${ }^{*} \mathrm{P}<0.05$ vs. the si-NC group. JARID2, protein Jumonji; si, small interfering RNA; NC, negative control; OD, optical density.

Matrigel invasion assay demonstrated that downregulation of JARID2 reduced the number of invasive SKOV3 cells when compared with the si-NC group (Fig. 3B).

Downregulation of JARID2 inhibits the epithelial-mesenchymal transition (EMT in ovarian cancer cells. To better investigate the effects of JARID2 on ovarian cancer cell migration and invasion, the expression of EMT-associated 

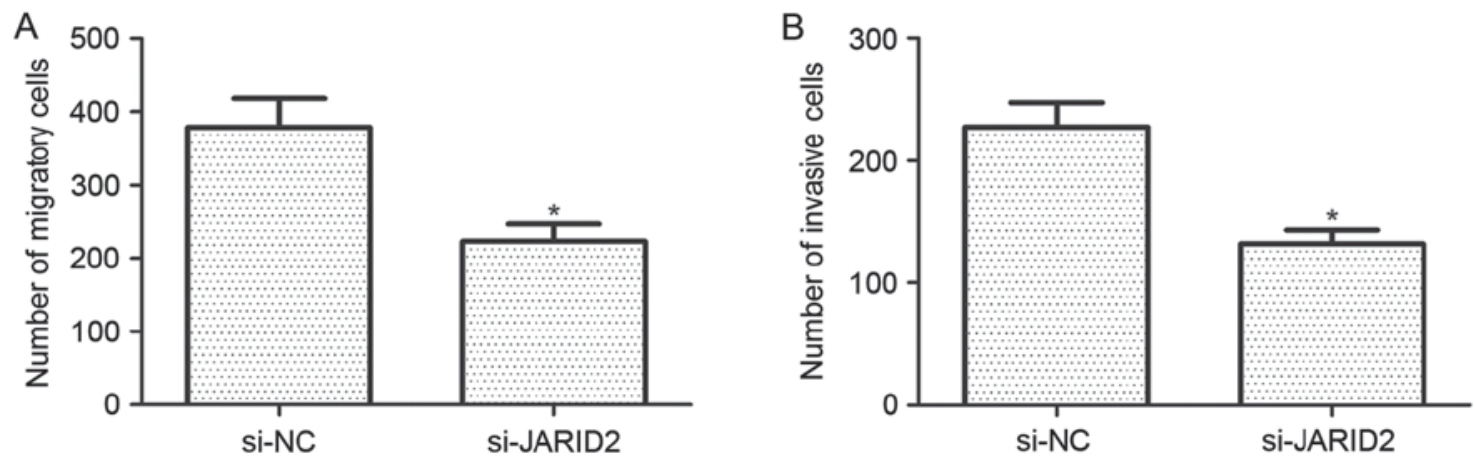

Figure 3. Downregulation of JARID2 inhibits the migration and invasion of ovarian cancer cells. SKOV3 cells were transfected with si-JARID2 or si-NC for $24 \mathrm{~h}$. (A) Cell migration was evaluated by Transwell migration assay. (B) Cell invasion was detected using the Matrigel invasion assay. All the experiments were repeated at least three times. " $\mathrm{P}<0.05$ vs. the si-NC group. JARID2, protein Jumonji; si; small interfering RNA; NC; negative control.
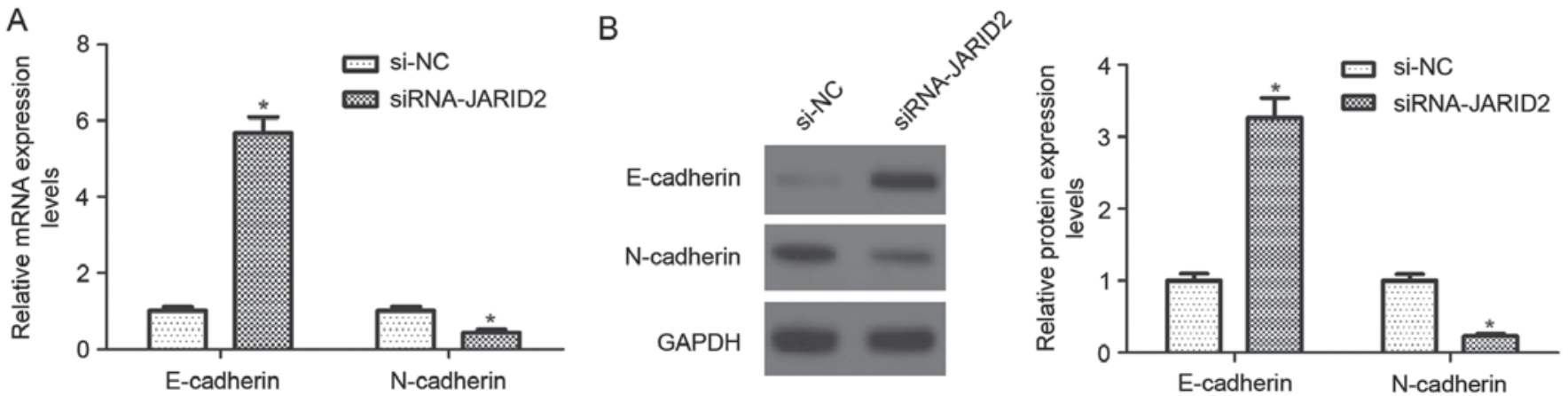

Figure 4. Downregulation of JARID2 inhibits epithelial-mesenchymal transition in ovarian cancer cells. SKOV3 cells were transfected with si-JARID2 or si-NC for $24 \mathrm{~h}$. (A) mRNA expression levels of E-cadherin and N-cadherin were evaluated by using the reverse transcription-quantitative polymerase chain reaction. (B) Protein expression levels of E-cadherin and N-cadherin were evaluated by western blotting. All the experiments were repeated at least three times. ${ }^{*} \mathrm{P}<0.05$ vs. the si-NC group. JARID2, protein Jumonji; si; small interfering RNA; NC; negative control; E, epithelial; N, neural.
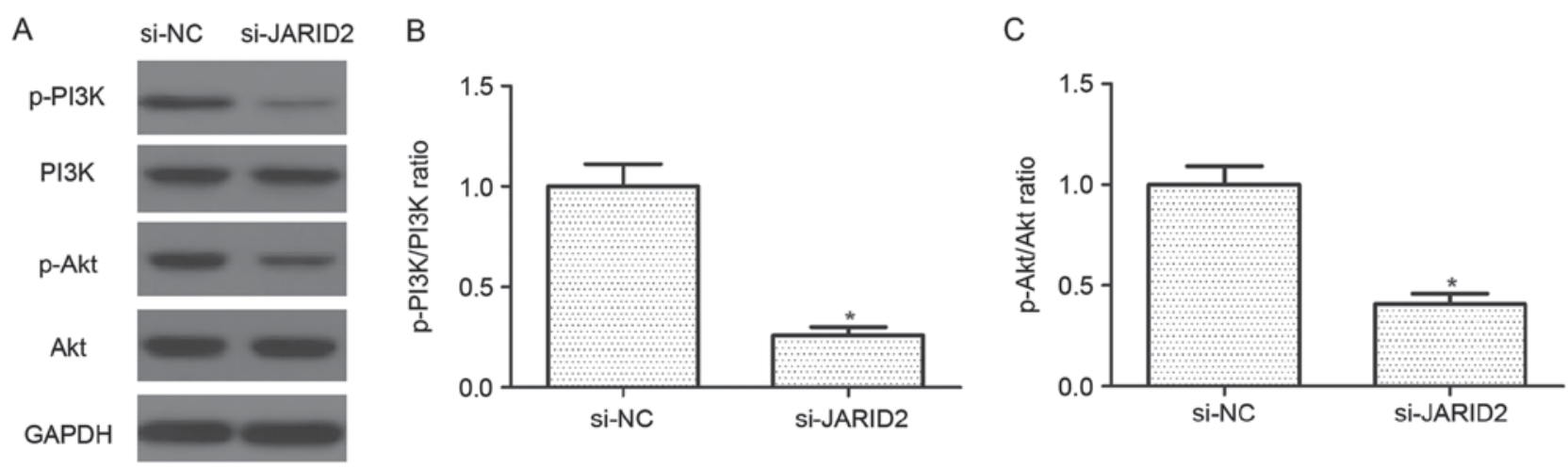

Figure 5. Downregulation of JARID2 inhibits the activation of the PI3K/Akt signaling pathway in ovarian cancer cells. SKOV3 cells were infected with si-JARID2 or si-NC for $24 \mathrm{~h}$. (A) Protein expression levels of PI3K, p-PI3K, Akt and p-Akt were evaluated by western blotting assay. Relative protein levels of (B) p-PI3K and (C) p-Akt were quantified using Image-Pro Plus 6.0 software. All the experiments were repeated at least three times. " $\mathrm{P}<0.05$ vs. the si-NC group. JARID2, protein Jumonji; si; small interfering RNA; NC; negative control; PI3K, phosphoinositide 3-kinase; Akt, protein kinase B; p, phosphorylated.

markers was evaluated using RT-qPCR and western blot analysis. As indicated in Fig. 4A, the mRNA expression of E-cadherin was significantly upregulated in SKOV3 cells transfected with si-JARID2, whereas, the mRNA expression of $\mathrm{N}$-cadherin was downregulated, compared with the si-NC group Similarly, downregulation of JARID2 caused a 3.2-fold increase in E-cadherin protein expression and a decrease in $\mathrm{N}$-cadherin protein expression in SKOV3 cells, as compared with the si-NC group (Fig. 4B).
Downregulation of JARID2 inhibits the activation of the PI3K/Akt signaling pathway in ovarian cancer cells. The PI3K/Akt signaling pathway serves an important role in the development and progression of ovarian cancer. Therefore, the effect of JARID2 on the activation of the PI3K/Akt signaling pathway was investigated in SKOV3 cells. As indicated in Fig. 5, knockdown of JARID2 suppressed the protein expression levels of p-PI3K and p-Akt in SKOV3 cells, as compared with the si-NC group. 


\section{Discussion}

In the present study, it was demonstrated that the expression of JARID2 is upregulated in human ovarian cancer cell lines. Furthermore, downregulation of JARID2 significantly suppressed the proliferation, migration, invasion and EMT in human ovarian cancer cells. Mechanistically, downregulation of JARID2 decreased the protein expression levels of p-PI3K and p-Akt in ovarian cancer cells.

Several studies reported that JARID2 serves a critical role in cancer development and progression. Walters et al (11) reported that JARID2 is overexpressed in rhabdomyosarcomas. Knockdown of JARID2 resulted in reduced cell proliferation coupled with increased myogenic differentiation (11). Another study confirmed that the expression of JARID2 was significantly upregulated in HCC tissues (14). In accordance with previous studies, in the current study, it was observed that JARID2 is expressed at high levels in human ovarian cancer cell lines. Downregulation of JARID2 significantly suppressed the proliferation of human ovarian cancer cells. These data suggested that JARID2 is involved in the carcinogenesis of ovarian cancer.

Ovarian cancer is the most lethal gynecological malignancy due to its high metastatic ability (16). The increased motility and invasiveness of malignant tumor cells is associated with the EMT phenotype. Ovarian cancer is characterized by the loss of epithelial differentiation and acquisition of mesenchymal-like cellular competence of tumor cells. During this process, epithelial cells downregulate the expression of cell adhesion molecules, including E-cadherin, dissolve cell-cell junctions, lose their apical-basal polarity, and enhance migratory and invasive properties $(17,18)$. In the present study, it was observed that downregulation of JARID2 suppressed the migration and invasion of ovarian cancer cells. In addition, it was observed that downregulation of JARID2 increased the expression of E-cadherin and decreased the expression of $\mathrm{N}$-cadherin in ovarian cancer cells. These results suggested that JARID2 may be an important contributor to EMT progression, thus facilitating the migration and invasion of ovarian cancer cells.

Increasing evidence has reported that the PI3K/Akt signaling pathway serves important roles in the progression of ovarian cancer (19-21). PI3K is activated by oncogenes, and activated PI3K promotes cancer cell growth and invasion (22). Akt, a downstream effector of PI3K, is implicated in various cellular processes, including cell proliferation, cell invasion, metabolism and EMT (23). It has been reported that the activation of Akt enhances EMT, downregulates E-cadherin transcription, and increases cancer cell migration and invasion (24). PI3K/Akt signaling pathway activation is associated with higher invasive and migratory capacities in human ovarian cancer cells (25). Therefore, inhibiting this pathway may be beneficial for the treatment of ovarian cancer. It was observed that knockdown of JARID2 downregulated the protein expression levels of p-PI3K and p-Akt in ovarian cancer cells. These results suggested that knockdown of JARID2 inhibits cell proliferation and migration through the inactivation of the PI3K/Akt signaling pathway.

In conclusion, the results of the present study demonstrate that JARID2 may serve important roles in ovarian cancer cell proliferation, invasion and EMT. Therefore, JARID2 may be a potential therapeutic target for the treatment of ovariancancer.

\section{Acknowledgements}

The present study was supported by the National Natural Science Foundation of China (grant no. 81402139).

\section{References}

1. Kuznia AL and Roett MA: Genital cancers in women: Ovarian cancer. FP Essent 438: 24-30, 2015.

2. Nicoletto MO, Artioli G, Donach M, Sileni VC, Monfardini S, Talamini R, Veronesi A, Ferrazzi E, Tumolo S, Visonà E, et al: Elderly ovarian cancer: Treatment with mitoxantrone-carboplatin. Gynecol Oncol 80: 221-226, 2001.

3. Rustin GJ, van der Burg ME, Griffin CL, Guthrie D, Lamont A, Jayson GC, Kristensen G, Mediola C, Coens C, Qian W, et al: Early versus delayed treatment of relapsed ovarian cancer (MRC OV05/EORTC 55955): A randomised trial. Lancet 376: 1155-1163, 2010.

4. Harries M and Gore M: Part II: Chemotherapy for epithelial ovarian cancer-treatment of recurrent disease. Lancet Oncol 3: 537-545, 2002.

5. Sundar S, Neal RD and Kehoe S: Diagnosis of ovarian cancer. BMJ 351: h4443, 2015.

6. Qian Q, Yan Y, Yang J, Cao D, Zhu Z, Wu M, Chen J, Lang J and Shen K: Management and prognosis of patients with ovarian sex cord tumor with annular tubules: A retrospective study. BMC Cancer 15: 270, 2015.

7. Shirato H, Ogawa S, Nakajima K, Inagawa $M$, Kojima $M$, Tachibana M, Shinkai Y and Takeuchi T: A jumonji (Jarid2) protein complex represses cyclin D1 expression by methylation of histone H3-K9. J Biol Chem 284: 733-739, 2009.

8. Sanulli S, Justin N, Teissandier A, Ancelin K, Portoso M, Caron M, Michaud A, Lombard B, da Rocha ST, Offer J, et al: Jarid2 Methylation via the PRC2 complex regulates H3K27me3 deposition during cell differentiation. Mol Cell 57: 769-783, 2015.

9. Landeira D, Sauer S, Poot R, Dvorkina M, Mazzarella L, Jørgensen HF, Pereira CF, Leleu M,Piccolo FM, Spivakov M, et al: Jarid 2 is a PRC2 component in embryonic stem cells required for multi-lineage differentiation and recruitment of PRC1 and RNA Polymerase II to developmental regulators. Nat Cell Biol 12: 618-624, 2010.

10. Peng JC, Valouev A, Swigut T, Zhang J, Zhao Y, Sidow A and Wysocka J: Jarid2/Jumonji coordinates control of PRC2 enzymatic activity and target gene occupancy in pluripotent cells. Cell 139: 1290-1302, 2009.

11. Walters ZS, Villarejo-Balcells B, Olmos D, Buist TW, Missiaglia E, Allen R, Al-lazikani B, Garrett MD, Blagg J and Shipley J: JARID2 is a direct target of the PAX3-FOXO1 fusion protein and inhibits myogenic differentiation of rhabdomyosarcoma cells. Oncogene 33: 1148-1157, 2014.

12. Tange S, Oktyabri D, Terashima M, Ishimura A and Suzuki T: JARID2 is involved in transforming growth factor-beta-induced epithelial-mesenchymal transition of lung and colon cancer cell lines. PLoS One 9: e115684, 2014.

13. Zhu Z, Tang LS, Xu L, Qin X, Mao S, Song Y, Liu L, Li F, Liu P, Yi L, et al: Genome-wide association study identifies new susceptibility loci for adolescent idiopathic scoliosis in Chinese girls. Nat Commun 6: 8355, 2015.

14. Lei X, Xu JF, Chang RM, Fang F, Zuo CH and Yang LY: JARID2 promotes invasion and metastasis of hepatocellular carcinoma by facilitating epithelial-mesenchymal transition through PTEN/AKT signaling. Oncotarget 7: 40266-40284, 2016.

15. Livak KJ and Schmittgen TD: Analysis of relative gene expression data using real-tie quantitative PCR and the 2(-Delta Delta C(T)) method. Methods 25: 402-408, 2001

16. Li L, Wang L, Zhang W, Tang B, Zhang J, Song H, Yao D, Tang Y, Chen X, Yang Z, et al: Correlation of serum VEGF levels with clinical stage, therapy efficacy, tumor metastasis and patient survival in ovarian cancer. Anticancer Res 24: 1973-1979, 2004.

17. De Craene B and Berx G: Regulatory networks defining EMT during cancer initiation and progression. Nat Rev Cancer 13: 97-110, 2013

18. Yilmaz M and Christofori G: EMT, the cytoskeleton, and cancer cell invasion. Cancer Metast Rev 28: 15-33, 2009. 
19. Meng Q, Xia C, Fang J, Rojanasakul Y and Jiang BH: Role of $\mathrm{PI} 3 \mathrm{~K}$ and AKT specific isoforms in ovarian cancer cell migration, invasion and proliferation through the p70S6K1 pathway. Cell Signal 18: 2262-2271, 2006.

20. Huang J, Zhang L, Greshock J, Colligon TA, Wang Y, Ward R, Katsaros D, Lassus H, Butzow R, Godwin AK, et al: Frequent genetic abnormalities of the PI3K/AKT pathway in primary ovarian cancer predict patient outcome. Genes Chromosomes Cancer 50: 606-618, 2011.

21. Lau MT and Leung PC: The PI3K/Akt/mTOR signaling pathway mediates insulin-like growth factor 1-induced E-cadherin down-regulation and cell proliferation in ovarian cancer cells. Cancer Lett 326: 191-198, 2012.

22. Luo J, Manning BD and Cantley LC: Targeting the PI3K-Akt pathway in human cancer: Rationale and promise. Cancer Cell 4 : 257-262, 2003.
23. Manning BD and Cantley LC: AKT/PKB signaling: Navigating downstream. Cell 129: 1261-1274, 2007.

24. Grille SJ, Bellacosa A, Upson J, Klein-Szanto AJ, van Roy F, Lee-Kwon W, Donowitz M, Tsichlis PN and Larue L: The protein kinase Akt induces epithelial mesenchymal transition and promotes enhanced motility and invasiveness of squamous cell carcinoma lines. Cancer Res 63: 2172-2178, 2003.

25. Bai H, Li H, Li W, Gui T, Yang J, Cao D and Shen K: The $\mathrm{PI} 3 \mathrm{~K} / \mathrm{AKT} / \mathrm{mTOR}$ pathway is a potential predictor of distinct invasive and migratory capacities in human ovarian cancer cell lines. Oncotarget 6: 25520-25532, 2015. 